\title{
Biopsia de mucosa nasal: descripción de técnicas y comparación de los resultados con gubia versus otros instrumentos en un hospital de referencia
}

\author{
Diana Patricia Crizón-Díazi; Victoria Eugenia Franco ²; Claudia Carolina Colmenares³
}

\section{RESUMEN}

Introducción: para la biopsia de mucosa nasal se usan diversos instrumentos, que pueden variar el resultado histológico. El propósito del estudio es describir y determinar la frecuencia de resultados histológicos satisfactorios de la biopsia tomada con gubia versus otros instrumentales en pacientes con lesiones de mucosa nasal.

Materiales y métodos: estudio de corte transversal, que incluyó registros clínicos de pacientes en quienes se realizó biopsia de mucosa nasal entre 2005 y 2016 en un hospital dermatológico en Bogotá, Colombia. Se excluyeron biopsias extrainstitucionales. Se analizaron características demográficas, clínicas y paraclínicas. El resultado satisfactorio se definió como la obtención de muestra suficiente sin presencia de artificios que impidiera al patólogo confirmar el diagnóstico. Adicionalmente, se reportó diámetro y profundidad de las muestras.

Resultados: se incluyeron 98 registros. En el 70,4\% de los casos se tomó biopsia con gubia. El diámetro de la muestra con gubia fue ligeramente mayor $(3,2 \mathrm{~mm}$ vs. $2,8 \mathrm{~mm}[p=0,16])$. La frecuencia de resultado satisfactorio fue similar en quienes se utilizó gubia $(94,2 \%)$ u otro instrumental $(93,1 \%)$. No obstante, las biopsias con resultado satisfactorio fueron de mayor diámetro y profundidad.

Discusión: no se observaron diferencias en la frecuencia de resultados satisfactorios de acuerdo con el tipo de instrumental; probablemente la calidad de la muestra es dependiente del operador. Para aumentar la probabilidad de un resultado satisfactorio se sugiere tener una muestra con diámetro mayor de 3 mm y mínimo 2 mm de profundidad.

PALABRAS Clave: Biopsia; Mucosa nasal; Procedimientos diagnósticos; Técnicas.

1. Posgrado de Dermatología, Fundación Universitaria Sanitas, Bogotá, Colombia. Hospital Universitario Centro Dermatológico Federico Lleras Acosta, Bogotá, Colombia. ORCID https://orcid.org/oooo-0o03-1878-30oX

2. Posgrado de Dermatología, Fundación Universitaria Sanitas, Bogotá, Colombia. Hospital Universitario Centro Dermatológico Federico Lleras Acosta, Bogotá, Colombia. ORCID https://orcid.org/oooo-ooo1-9463-7448

3. Unidad de investigación, Fundación Universitaria Sanitas, Bogotá, Colombia. ORCID https://orcid.org/oooo-oo03-1847-4282

Correspondencia: Diana Patricia Crizón-Díaz; email: dianac4d@gmail.com

Recibido: 25/03/20; aceptado: 25/04/20

Cómo citar: Crizón, DP; Franco, VE; Colmenares, CC. Biopsia de mucosa nasal: descripción de técnicas y comparación de los resultados con gubia versus otros instrumentos en un hospital de referencia. Rev Asoc Colomb Dermatol. Vol 28(4): octubre - diciembre, 2020, 308-317. DOI: https://doi.org/10.29176/2590843X.1550

Financiación: ninguna, conflictos de interés: ninguno 


\section{NASAL MUCOSA BIOPSY: DESCRIPTION OF TECHNIQUES AND COMPARISON OF RESULTS WITH GOUGE VERSUS OTHER INSTRUMENTS IN A REFERENCE HOSPITAL}

\section{SUMMARY}

Introduction: For the nasal mucosa biopsy, various instruments are used, and the histological result may vary. The purpose of this study is to describe and determine the frequency of satisfactory histological results of biopsy taken with gouge versus other instruments in patients with nasal mucosal lesions.

Materials and methods: Cross-sectional study; included clinical records of patients who underwent nasal mucosa biopsy between 2005 to 2016 in a dermatological hospital in Bogotá, Colombia. Extra-institutional biopsies were excluded. Demographic, clinical and paraclinical characteristics were analyzed. The satisfactory result was defined as obtaining a sufficient sample without the presence of artifices that can prevent the pathologist from confirming the diagnosis. Additionally, diameter and depth of samples were reported.

Results: 98 records were included. In $70.4 \%$ of the cases biopsy was taken with gouge. The diameter of the sample with gouge was slightly larger $(3.2 \mathrm{~mm}$ vs $2.8 \mathrm{~mm}[\mathrm{p}=0.16])$. The frequency of satisfactory results was similar in those who used gouge (94.2\%) or other instruments (93.1\%). However, the biopsies with satisfactory results were of greater diameter and depth.

Discussion: No differences were observed in the frequency of satisfactory results according to the type of instruments, probably the quality of the sample is operator dependent. To increase the probability of a satisfactory result, it is suggested to have a sample with a diameter greater than three mm and at least two mm deep.

KEY WORDS: Biopsy; Diagnostic; Nasal mucosa; Procedures; Techniques.

\section{INTRODUCCIÓN}

Existen enfermedades que afectan la mucosa nasal entre las que se encuentran las infecciones por protozoos, como la leishmaniasis ${ }^{(1,2)}$; las infecciones bacterianas, como la sífilis, la rinoescleroma (3), la tuberculosis nasal y la lepra ${ }^{(4,5)}$; las enfermedades por hongos, como la mucormicosis, la conidiobolomicosis, la rinosporidiosis, las aspergilosis y la histoplasmosis ${ }^{(6,7)}$; las enfermedades inflamatorias, como la sarcoidosis nasal, la granulomatosis rinogénica en la granulomatosis de Wegener; y las enfermedades tumorales, como el linfoma $\mathrm{NK}^{(8,9)}$.

Estas enfermedades pueden tener presentaciones clínicas similares y la detección tardía puede generar secuelas como deformidades nasales, con un impacto negativo en la calidad de vida. Por eso, el diagnóstico debe ser oportuno y correcto, de allí la importancia de la biopsia de tejido intranasal para confirmar el diagnóstico.
La biopsia de mucosa nasal puede ser realizada por diferentes profesionales de la Medicina, con una variedad de instrumentos como tijera nasal, bisturí, punch, cureta y gubia nasal (figura 1) ${ }^{(10)}$; además, se requiere un quirófano y anestesia local o general ${ }^{(3)}$.

Para la biopsia con tijera nasal se realizan cortes de la lesión, con lo cual se puede extraer una muestra insuficiente para el estudio. En la técnica con bisturí se realiza una elipse y el trazo debe ser constante y no a secciones para evitar las laceraciones en los bordes ${ }^{(11,12)}$. El punch es un instrumento con una cuchilla circular, que se penetra hasta el tejido subcutáneo para obtener un cilindro de la muestra ${ }^{(13)}$. Para la biopsia con $\mathrm{cu}^{-}$ reta existen varios tipos, identificados por tamaño del o al 8 de acuerdo con el diámetro del eje más largo en milímetros. La muestra debe ser removida colocando el borde cortante de la cureta con un movimiento horizontal $^{(14)}$; finalmente, la técnica con gubia nasal es un tipo especial de pinza con bordes cortantes. El ángulo de la punta permite cortar tejidos en áreas difíciles de $\operatorname{alcanzar}^{(10)}$. 


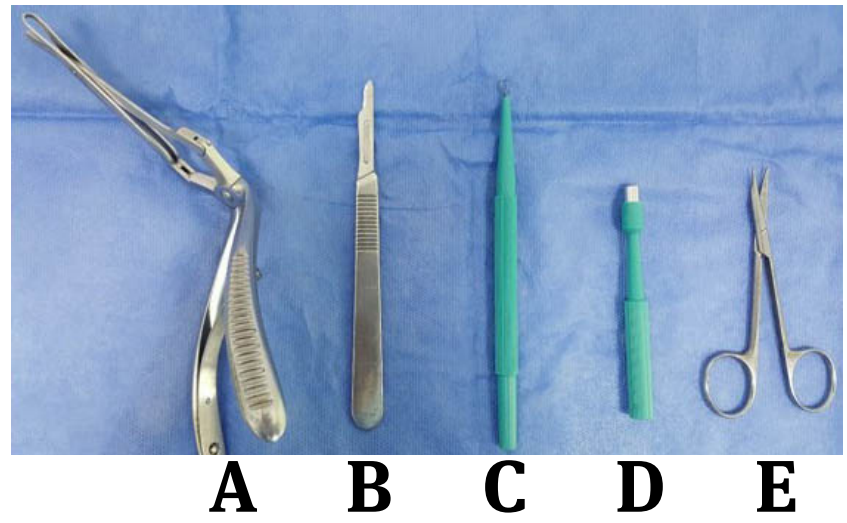

Figura 1. Instrumentos para realizar biopsia de mucosa nasal. A) Gubia. B) Bisturí. C) Cureta. D) Punch. E) Tijeras.

Dado que no hay unanimidad en cuanto a la técnica para la toma de la muestra, es importante describir los resultados obtenidos con los instrumentos utilizados en un centro de referencia, además de evaluar tales instrumentos según las diferentes patologías que afectan la mucosa nasal, para conocer de forma objetiva aquellas que tengan mayor probabilidad de éxito por tener material suficiente y reducir así la frecuencia de reintervención y error en el diagnóstico por material insuficiente.

En el Hospital Universitario Centro Dermatológico Federico Lleras Acosta, un centro dermatológico de referencia en Bogotá, Colombia, se realizan alrededor de diez biopsias de mucosa nasal por año, por lo que este trabajo busca describir y determinar la frecuencia de resultados histológicos satisfactorios de la biopsia tomada con gubia versus otros instrumentales en pacientes con lesiones de mucosa nasal atendidos en el Hospital en el período comprendido entre 2005 y 2016.

\section{MATERIAL Y METODOLOGÍA}

Estudio observacional, analítico, de corte transversal y retrospectivo, realizado en el Hospital Universitario Centro Dermatológico Federico Lleras Acosta en la ciudad de Bogotá, Colombia, durante los años 2005 a 2016.

Se incluyeron pacientes en los que se realizaron biopsias de mucosa nasal para el diagnóstico de lesiones que comprometieran dicha zona y que tuvieran el reporte de histopatología registrado en la historia clínica. Se excluyeron aquellos pacientes con historia clínica incompleta sobre el examen físico y a los que se realizaron biopsias extrainstitucionales.

Se revisaron historias clínicas para recolectar la información relacionada con edad, sexo, residencia, ocupación, tiempo de evolución en meses, indicación de la biopsia (diagnóstico clínico), presencia de costras y perforación septal, cantidad de sangrado (escaso: cede con maniobras de presión digital y mínima o nula cauterización química; moderado: requiere un pseudotaponamiento; y abundante: requiere taponamiento nasal anterior y/o posterior), instrumental usado, resultado confirmatorio histopatológico, nueva biopsia, muestra suficiente (mínimo de $4 \mathrm{~mm}$ de diámetro y profundidad de $3 \mathrm{~mm}$ ), tamaño de biopsia (diámetro y profundidad en milímetros), resultado satisfactorio (obtención de muestra suficiente, sin presencia de costras, sangre $\mathrm{u}$ otro artificio que impidiera al patólogo confirmar el diagnóstico de lesiones de la mucosa nasal). Los sujetos incluidos se dividieron en dos grupos: el primero, pacientes en los que se realizó biopsia de mucosa nasal con gubia, y el segundo, pacientes en quienes se utilizaron otros instrumentos. Otros instrumentos incluyeron: bisturí, cureta, punch y tijeras.

Inicialmente se realizó un análisis descriptivo de la información recolectada. Las variables cualitativas se describen como frecuencias absolutas y relativas. Las variables cuantitativas se reportan a través de medidas de tendencia central y de dispersión según correspondiera. Para establecer diferencias en la frecuencia de resultado satisfactorio (desenlace principal), así como los desenlaces secundarios, se utilizó la prueba de $\mathrm{X}^{2} \mathrm{o}$ Fisher para variables demográficas y clínicas cualitativas y la prueba de $t$ student o Wilcoxon para variables cuantitativas según correspondiese. Se interpretaron como significativas las diferencias con una $p<0,05$ con 
prueba de hipótesis a dos colas. El análisis se realizó en Stata $13.0^{\left({ }^{(15)}\right.}$. El presente estudio fue aprobado por el comité de ética en investigación del centro de referencia.

\section{RESULTADOS}

Se identificaron 256 pacientes con diagnóstico inicial de lesiones en mucosa nasal y que tenían orden de biopsia de nariz. Se excluyeron 158 pacientes. Finalmente, se incluyeron 98 pacientes que cumplían con los criterios de selección (figura 2).

La edad promedio fue de 51,2 años y la mayoría correspondió a población del género masculino $(60,2 \%)$. Una cuarta parte de los pacientes vivían en la capital del país (Bogotá), pero durante el período de interés también se atendieron pacientes de zonas selváticas. La ocupación de los pacientes fue variada. La principal indicación de biopsia de mucosa nasal fue la sospecha de leishmaniasis mucosa (tabla 1). La mediana del tiempo de evolución de la lesión fue de 12 meses y el hallazgo clínico más frecuente fue la infiltración de mucosa $(79,59 \%)$, seguido de perforación septal (50\%), eritema de la mucosa $(12,24 \%)$, úlcera $(10,20 \%)$, placa $(7,14 \%)$ y deformidad nasal $(3,06 \%)$.

El instrumento más utilizado para realizar biopsias de mucosa nasal fue la gubia, con un 70,41\% (69 biopsias), seguido de bisturí, con un 10,2 \% (10 biopsias), punch, con un 10,2 \% (10 biopsias), tijeras, con un 5,1\% ( 5 biopsias), bisturí y gubia, con un 3,06\% (3 biopsias), y cureta, con un 1,02\% (1 biopsia).

Con respecto a las características de la biopsia (tabla 2), en todas las muestras el diámetro promedio fue superior de $3 \mathrm{~mm}$. Así mismo, la mayoría de las biopsias presentaron sangrado escaso y no fue frecuente la presencia de costras. Sin embargo, para las biopsias que fueron tomadas con gubia, la mayoría tuvo un promedio de diámetro ligeramente mayor frente a las tomadas con otros instrumentos, con una diferencia de diámetro de 0,4 mm a favor del grupo de la gubia. En el caso de la profundidad, en ambos grupos la mediana de diámetro fue de $2 \mathrm{~mm}(p=0,04)$, aunque se tomó biopsia de tejidos hasta de $6 \mathrm{~mm}$ de profundidad en
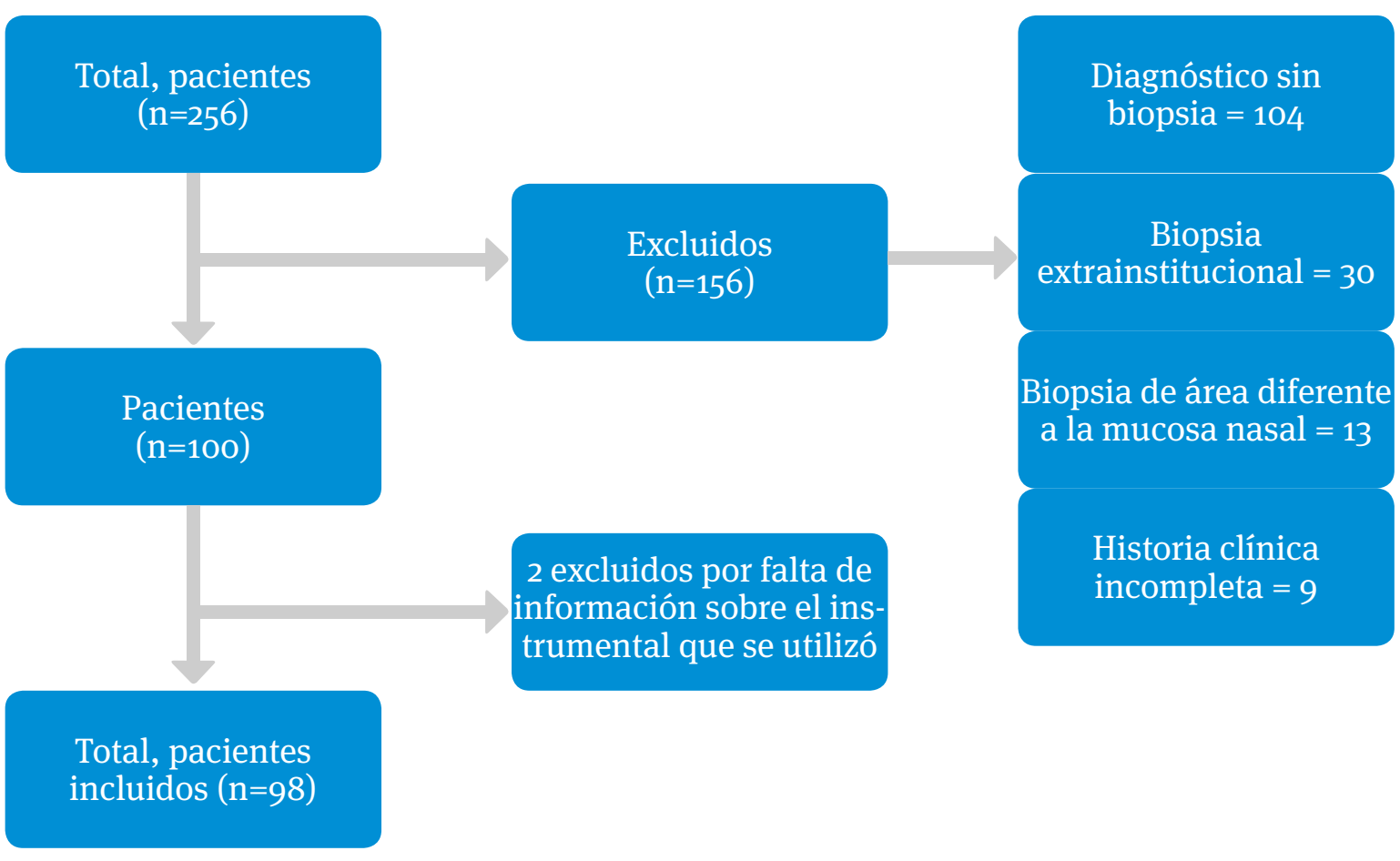

Historia clínica

incompleta = 9

Figura 2. Diagrama de flujo de la selección de los pacientes. 
Tabla 1. Características de la población e indicación de biopsia.

\begin{tabular}{|c|c|}
\hline Variable & $\begin{array}{l}\text { Todos } \\
n=98 \\
n(\%)\end{array}$ \\
\hline Edad* (años) & $51,2(19,9)$ \\
\hline \multicolumn{2}{|l|}{ Género } \\
\hline Masculino & $59(60,2)$ \\
\hline Femenino & $39(39,8)$ \\
\hline \multicolumn{2}{|l|}{ Residencia } \\
\hline Bogotá & $25(25,51)$ \\
\hline Cundinamarca & $23(23,47)$ \\
\hline Santander & $10(10,20)$ \\
\hline Meta & $8(8,16)$ \\
\hline Tolima & $7(7,14)$ \\
\hline Boyacá & $6(6,12)$ \\
\hline Caquetá & $6(6,12)$ \\
\hline Vichada & $3(3,06)$ \\
\hline Caldas & $2(2,04)$ \\
\hline Casanare & $2(2,04)$ \\
\hline Guaviare & $2(2,04)$ \\
\hline Bolívar & $1(1,02)$ \\
\hline Chocó & $1(1,02)$ \\
\hline Huila & $1(1,02)$ \\
\hline Vaupés & $1(1,02)$ \\
\hline \multicolumn{2}{|l|}{ Ocupación } \\
\hline No reporta/ desempleado & $43(43,88)$ \\
\hline Hogar & $13(27,0)$ \\
\hline Otros & $11(11,22)$ \\
\hline No registra & $9(9,18)$ \\
\hline Educación & $7(7,14)$ \\
\hline Construcción e industria & $5(5,10)$ \\
\hline Administrativo/oficina & $4(4,08)$ \\
\hline Pensionado & $3(3,06)$ \\
\hline Cocina & $2(2,04)$ \\
\hline Militar & $1(1,02)$ \\
\hline \multicolumn{2}{|l|}{ Indicación de biopsia } \\
\hline Leishmaniasis mucosa & $87(88,78)$ \\
\hline Perforación septal & $4(4,08)$ \\
\hline Tumor de la línea media ${ }^{\dagger}$ & $3(3,06)$ \\
\hline Lepra lepromatosa & $1(1,02)$ \\
\hline Inflamación secundaria por consumo de cocaína ${ }^{\dagger}$ & $1(1,02)$ \\
\hline Lepra $^{\dagger}$ & $1(1,02)$ \\
\hline Paracoccidioidomicosis ${ }^{\dagger}$ & $1(1,02)$ \\
\hline
\end{tabular}

^Promedio (DE) (Desviación Estandar); † Diagnóstico diferencial. 
Tabla 2. Características de la muestra obtenida.

\begin{tabular}{|c|c|c|c|c|}
\hline Variable & $\begin{array}{l}n(\%) \\
n=98\end{array}$ & $\begin{array}{l}\text { Gubia } \\
n=69\end{array}$ & $\begin{array}{l}\text { Otro instrumental } \\
\qquad n=29\end{array}$ & $p$ \\
\hline Diámetro* $(\mathrm{mm})$ & $3,1(1,0)$ & $3,2(0,9)$ & $2,8(1,0)$ & 0,16 \\
\hline Profundidad $^{\dagger}(\mathrm{mm})$ & $2(2-3)$ & $2(2-3)$ & $2(2-3)$ & 0,04 \\
\hline \multicolumn{5}{|l|}{ Costras } \\
\hline Sí & $30(30,61)$ & $21(30,4)$ & $9(31,0)$ & 0,95 \\
\hline No & $68(69,39)$ & $48(69,6)$ & $20(69,0)$ & \\
\hline \multicolumn{5}{|l|}{ Sangrado } \\
\hline Escaso & $92(92,88)$ & $63(91,3)$ & 29 (100) & 0,17 \\
\hline Moderado & $6(6,12)$ & $6(8,7)$ & - & \\
\hline $\begin{array}{l}\text { Resultado } \\
\text { satisfactorio }\end{array}$ & $92(93,8)$ & $65(94,2)$ & $27(93,1)$ & 1 \\
\hline
\end{tabular}

*Promedio (DE); †mediana (RIQ) (Rango intercuartílico).

Para estimar las diferencias entre gubia vs otros instrumental de acuerdo al diámetro se utilizó la prueba t student.

Para estimar las diferencias entre gubia vs otros instrumental de acuerdo a la profundidad se utilizó la prueba de Wilcoxon.

Para estimar las diferencias entre gubia vs otros instrumental de acuerdo a la presencia de costras y resultado satisfactorio se utilizó la prueba de Chi ${ }^{2}$

Para estimar las diferencias entre gubia vs otros instrumental de acuerdo a la presencia de sangrado se utilizó el test exacto de Fischer

el grupo de toma con gubia (figura 3). Casi el 70\% de las muestras, tanto las tomadas con gubias, como las tomadas con otros instrumentales, no presentaron costras $(p=0,95)$ y el sangrado fue reportado como escaso en más del $92 \%$ de las muestras tomadas con gubias $\mathrm{y}$ en todas las muestras que se realizaron con otros instrumentales $(p=0,17)$. Por otra parte, el 94,90\% (93 casos) obtuvo muestra suficiente para el estudio histopatológico. El principal diagnóstico histológico reportado fue leishmaniasis mucosa (51\%), aunque también se informaron otras causas infecciosas y tumorales, además de algunos resultados que fueron no concluyentes (figura 4).

En relación con el resultado, solo 6 pacientes $(6,12 \%)$ no tuvieron resultado satisfactorio (aunque en un solo paciente la muestra se había reportado como suficiente).

La frecuencia de resultado satisfactorio fue similar en quienes se utilizó gubia en comparación con otro instrumental para la toma de la muestra $(\mathrm{n}=65[94,2 \%] v s$. $\mathrm{n}=27[93.1 \%] ; p=1,00)$.

Al comparar el tipo de resultado, respecto a las características del procedimiento, no se encontraron diferencias según el tipo de instrumental, presencia de costras o sangrado (tabla 3).

En relación con las características de la muestra y el resultado, el promedio del diámetro y la mediana de la profundidad fueron mayores en las muestras, con 

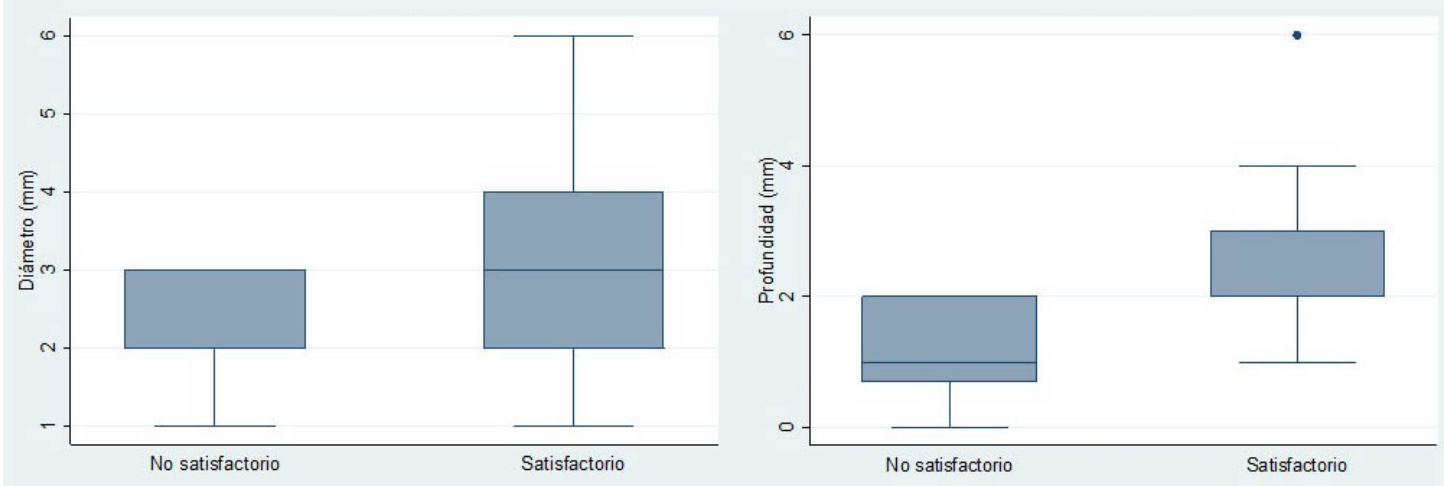

Figura 3. Diámetro y profundidad por resultado. (Esta es una figura descriptiva con el objetivo de comparar hallazgos, mas no estimar diferencias).

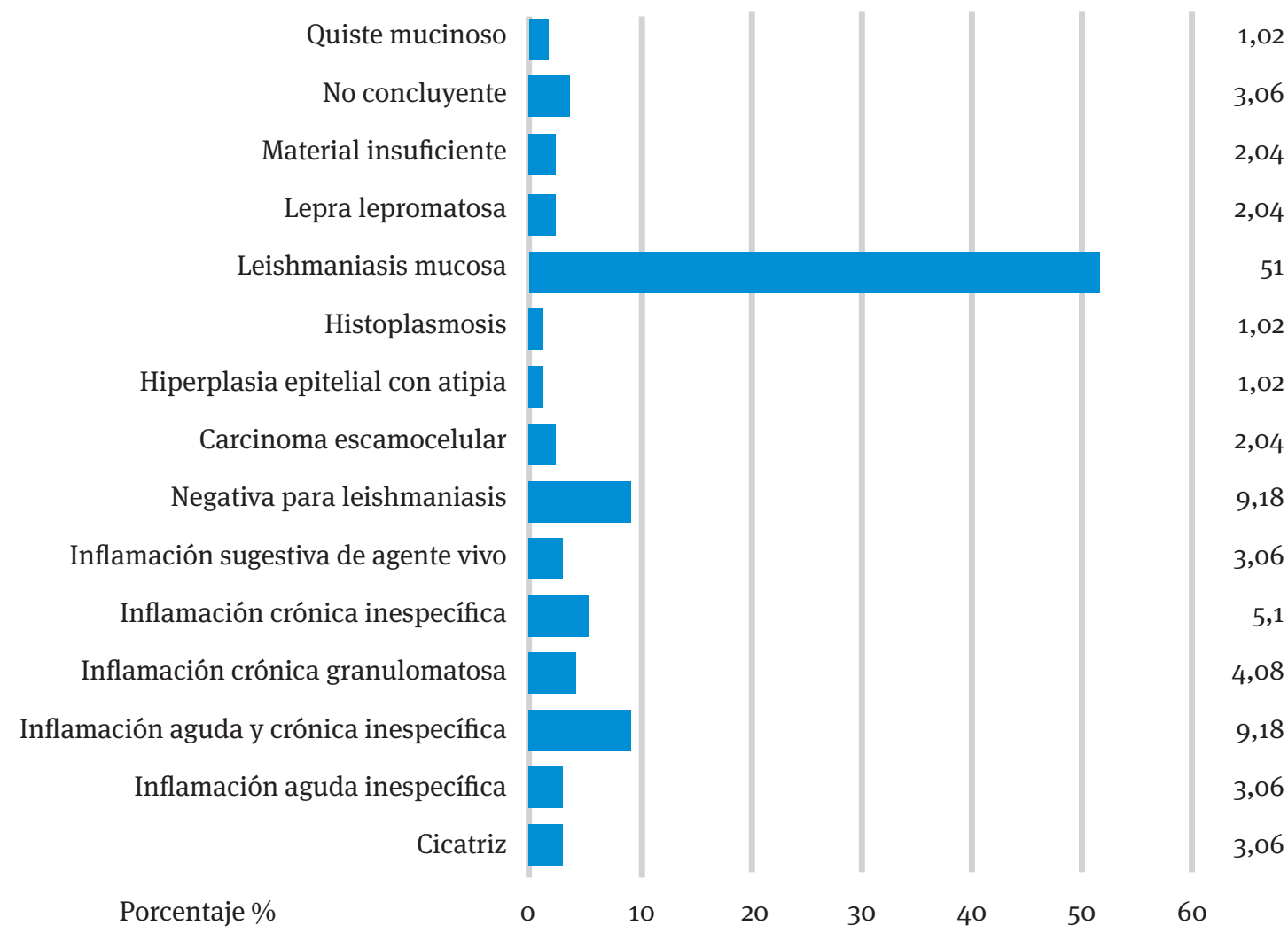

Figura 4. Distribución según los resultados histológicos.

resultado satisfactorio. Se presentó una diferencia de $1 \mathrm{~mm}$ para el diámetro entre el resultado satisfactorio (3,17 mm; desviación estándar [DE]: 1,00) y no satisfactorio (2,16 mm; DE: 0,75), siendo estadísticamente significativo ( $p=0,0171)$. En el caso de la profundidad, fue más frecuente encontrar muestras superficiales (1 mm [rango intercuartílico]; RIQ: 0,7-2 mm) en el grupo de resultado no satisfactorio que en el de resultado satisfactorio (2 [RIQ: 2-3 mm]; $p=0,0017$ ).
De los 98 pacientes en los que se realizó biopsia de la mucosa nasal, 4 pacientes $(4,08 \%)$ requirieron una segunda biopsia. En todos los casos, se obtuvo muestra suficiente durante la nueva biopsia. Los instrumentos usados para realizarlas fueron: gubia, en 2 pacientes (50\%), seguido de cureta, en 1 paciente (25\%), y tijera, en 1 paciente (25\%). La mediana del diámetro de las nuevas biopsias fue de 3,5 mm (RIQ: $3-5 \mathrm{~mm}$ ) y la mediana de la profundidad fue de $2 \mathrm{~mm}$ (RIQ: 1,5-2,5 $\mathrm{mm}$ ). 
Tabla 3. Características del procedimiento y muestra según resultado

\begin{tabular}{|c|c|c|c|}
\hline Variable & $\begin{array}{l}\text { Resultado } \\
\text { satisfactorio } \\
\qquad \begin{array}{c}n=92 \\
n(\%)\end{array}\end{array}$ & $\begin{array}{c}\text { Resultado no } \\
\text { satisfactorio } \\
n=6 \\
n(\%)\end{array}$ & $p^{*}$ \\
\hline \multicolumn{4}{|l|}{ Instrumental } \\
\hline Gubia y bisturí & $3(3,26)$ & $o(0)$ & \\
\hline Gubia & $65(70,65)$ & $4(66,67)$ & \\
\hline Bisturí & $9(9,78)$ & $1(16,67)$ & 0,537 \\
\hline Cureta & $1(1,09)$ & o (o) & \\
\hline Punch & $10(10,87)$ & $o(0)$ & \\
\hline Tijeras & $4(4,35)$ & $1(16,67)$ & \\
\hline \multicolumn{4}{|l|}{ Costras } \\
\hline Sí & $29(31,52)$ & $1(16,67)$ & 0,663 \\
\hline No & $63(68,48)$ & $5(83.33)$ & \\
\hline \multicolumn{4}{|l|}{ Sangrado } \\
\hline Escaso & $86(93,48)$ & $6(100)$ & 1 \\
\hline Moderado & $6(6,52)$ & $0(0,00)$ & \\
\hline
\end{tabular}

^Diferencias estimadas con la prueba exacta de Fisher.

\section{Puntos clave}

- Existen varias enfermedades que afectan la mucosa nasal y pueden tener similitud clínica.

- En la patología intranasal para un diagnóstico oportuno y correcto es útil la biopsia de mucosa nasal.

- Para la biopsia de mucosa nasal se usan diversos instrumentos, que pueden hacer variar el resultado histológico.

- Al evaluar varios instrumentos según las diferentes patologías que afectan la mucosa nasal, se puede conocer de forma objetiva aquellas que tengan mayor probabilidad de éxito por tener material suficiente y reducir así la frecuencia de reintervención y error en el diagnóstico por material insuficiente.

- Estudio realizado en hospital dermatológico de referencia, comparando gubia vs otros instrumentos.

- Estudio de corte transversal, que incluyó registros clínicos de pacientes en quienes se realizó biopsia de mucosa nasal en un periodo de 12 años ( 2005 -2016)

- $\quad$ Para aumentar la probabilidad de un resultado satisfactorio se sugiere tener una muestra con diámetro mayor de $3 \mathrm{~mm}$ y mínimo $2 \mathrm{~mm}$ de profundidad. 
En el 50\% los resultados histológicos fueron un diagnóstico definitivo; el resto se reportaron con patrón de inflamación aguda (25\%) y crónica (25\%). Al comparar el diámetro de las biopsias (inicial y nueva biopsia) en los 4 pacientes en quienes se repitió la biopsia, la mediana del diámetro en la biopsia inicial fue de 4 mm (RIQ: 3,5-4), ligeramente mayor comparada con la nueva biopsia, donde la mediana fue de $3,5 \mathrm{~mm}$ (RIQ: 3-5). Por otro lado, al comparar la profundidad de las biopsias (inicial y nueva biopsia) en los 4 pacientes en quienes se repitió la biopsia, la mediana de la profundidad en la biopsia inicial fue de $2 \mathrm{~mm}(1,5-2)$, muy similar comparada con la nueva biopsia, donde la mediana fue $2 \mathrm{~mm}(1,5-2,5)$.

Finalmente, en 5 pacientes se obtuvo muestra insuficiente, pero en ninguno se realizó biopsia nuevamente.

\section{DISCUSIÓN}

Se presentan los resultados de un estudio de pacientes con lesiones que comprometían la mucosa nasal y en quienes se realizó biopsia para evaluar la frecuencia de resultado histológico satisfactorio de la biopsia tomada con gubia versus otros instrumentales en un centro de atención de referencia en Bogotá, Colombia, con un período de observación de 12 años. Aunque el instrumento más usado para la toma de biopsia de mucosa nasal fue la gubia, probablemente por su disponibilidad en este centro, no se observaron diferencias en la frecuencia de resultados satisfactorios de acuerdo con el tipo de instrumental, lo cual puede corresponder a que la calidad de la muestra es dependiente del operador. Así mismo, se encontró que las muestras más superficiales y pequeñas tenían más frecuencia de resultados no satisfactorios, probablemente debido a que la escasa cantidad de tejido limitaba la correcta lectura y el diagnóstico por parte del médico patólogo.

En la literatura revisada solo se encontraron dos estudios similares. Prior y colaboradores (3) analizaron dos instrumentos: fórceps fenestrados versus tijeras Gerritsma en 160 pacientes, con el objetivo de evaluar la calidad de la muestra y las complicaciones con los distintos instrumentales. Se tomaron biopsias de 3 a 4 $\mathrm{mm}$ de diámetro. Fue necesario reintroducir en 6 ocasiones (2\%) la pinza de biopsia en la nariz debido a la poca muestra obtenida (98\% de éxito) ${ }^{(3)}$. En nuestro estudio, se encontró que en los pacientes con resultado satisfactorio la muestra tenía un diámetro mayor de 3 $\mathrm{mm}$, con una profundidad mínima de $2 \mathrm{~mm}$, rango si- milar al usado en el estudio de Prior y colaboradores; así mismo, el porcentaje de resultado satisfactorio fue similar al reportado por este autor.

En el estudio de Segal y colaboradores, se compararon dos técnicas para biopsia nasal en 46 pacientes, para determinar si existían diferencias entre el resultado patológico de la biopsia nasal y el resultado de la patología posquirúrgica, según el instrumental usado ${ }^{(11)}$. La biopsia se realizó en dos técnicas: la primera fue tomada con fórceps nasales directamente de la lesión y la segunda muestra fue tomada con tijeras nasal cortando el tejido y luego retirando la muestra. No se registró el diámetro ni la profundidad de la muestra. Los autores concluyeron que lo que explica las diferencias entre el resultado patológico de la biopsia nasal y el resultado de la patología posquirúrgica es la técnica usada para tomar la biopsia, ya que los fórceps crean artificios por aplastamiento, que se podrían reducir si se usa un método más delicado para las biopsias, como lo son las tijeras nasales ${ }^{(16)}$.

Con respecto al sangrado, en este estudio se encontró que más del 90\% de los pacientes presentaron sangrado escaso, incluso todos aquellos con resultado no satisfactorio; sin embargo, el sangrado ocurrió posterior a la toma de la muestra. Este mismo escenario fue reportado en el estudio de Prior y colaboradores, donde el sangrado fue clasificado como complicación del procedimiento, aunque no influyó en el resultado.

En este estudio, en cerca del 70\% de los pacientes, la muestra fue tomada con gubia, por lo que no fue posible establecer diferencias en el resultado de acuerdo con el instrumental utilizado, aunque es importante enfocarse en el tamaño y la profundidad de la muestra para obtener resultados satisfactorios y disminuir así la necesidad de realizar nuevas biopsias.

En los casos de pacientes que tuvieron muestra insuficiente y no se les hizo nueva biopsia, el diagnostico se confirmó por medio de inmunofluorescencia indirecta (estos tenían sospecha de leishmaniasis mucosa) y clínica, o en otros casos hubo pérdida del paciente, lo cual limitó su seguimiento y evolución. Por otra parte, a pesar de haberse realizado nuevas biopsias, solo el $50 \%$ de estos pacientes tuvo un diagnóstico definitivo. Es probable que los casos reportados como inflamación aguda y crónica correspondan a algún patrón no claro de enfermedad.

La biopsia de mucosa nasal es un examen útil cuando se sospecha la posibilidad de enfermedad infecciosa, 
inflamatoria o tumoral como responsable de los síntomas. Las patologías pueden exhibir presentaciones clínicas similares, pero debido a que existen varios instrumentos y técnicas para realizar la biopsia, puede tener limitaciones y variaciones en su resultado. Esto lleva a informes de patología reportados como muestra insuficiente, por lo que existe la necesidad de evaluar de manera objetiva la mejor técnica e instrumental para tener una herramienta con la menor morbilidad y limitaciones posibles para el diagnóstico de las enfermedades que afectan la mucosa nasal.

Como fortalezas de este estudio se resalta el haber sido realizado en un centro de referencia en dermatología, con un número importante de pacientes con diagnósticos de patologías que con frecuencia pueden afectar la mucosa nasal. Se analizó la información correspondiente a un período de 12 años, lo cual permitió evaluar varios instrumentos utilizados para tomar biopsias de mucosa nasal. Por tratarse de un estudio retrospectivo, hay algunas limitaciones, como la ausencia de datos para algunas de las variables, especialmente datos del examen físico, que al momento del registro no fueron detallados.

\section{CONCLUSIONES}

Las enfermedades que comprometen la mucosa nasal pueden ser similares clínicamente y la biopsia de mucosa nasal es una herramienta útil para confirmar el diagnóstico. Para aumentar la probabilidad de un resultado satisfactorio, se sugiere tener una muestra suficiente con diámetro mayor de $3 \mathrm{~mm}$ y mínimo de $2 \mathrm{~mm}$ de profundidad. Los resultados de este estudio son útiles para realizar nuevos estudios de carácter prospectivo donde se evalúen diversos instrumentales para la toma de biopsia de mucosa nasal y determinar si existen diferencias para obtener resultados satisfactorios.

\section{REFERENCIAS}

1. Oddone R, Arbo C, Nara E, Velázquez G, Acosta $\mathrm{M}$, Poletti D, et al. Utilidad diagnóstica de los métodos laboratoriales en leishmaniasis mucosa, incluyendo la PCR. Not Tec Lab. 2004;12(4):7-9.

2. Gul HC, Tosun F, Karakas A, Koru O, Onguru O, Mert $\mathrm{G}$, et al. A case of mucosal leishmaniasis: Mimicking intranasal tumor with perforation of septum. J Microbiol Immunol Infect. 2016;49(4):604-7. doi: 10.1016/j.jmii.2013.11.007
3. Prior AJ, Calderon MA, Lavelle RJ, Davies RJ. Nasal biopsy: indications, techniques and complications. Respir Med. 1995;89(3):161-9. doi: 10.1016/0954-6111(95)90242-2

4. Rodriguez G, Pinto R. La lepra. Imágenes y conceptos. Medellín: Editorial Universidad de Antioquia; 2007.

5. Truman R, Krahenbuhl J. Viable M. leprae as a research reagent. Int J Lepr Other Mycobact Dis. 2001;69(1):1-12.

6. Milanes R, Caro C, Vélez C, Marrugo O. Diagnóstico diferencial de la rinosporidiosis: a propósito de un caso. Iatreia. 2012;25(3):272-6.

7. Jaimes A, Muvdi S, Alvarado Z, Rodríguez G. Perforation of the nasal septum as the first sign of histoplasmosis associated with AIDS and review of published literature. Mycopathologia. 2013;176(12):145-50. doi: 10.1007/s11046-013-9662-z

8. Reyes HD, Marquez RE, Fuste JC, Ramos VJ. Granuloma letal de la línea media como diagnóstico diferencial de la vasculitis de Wegener. Presentación de una paciente. Acta Med Centro. 2015;9(2):34-40.

9. Mallya V, Singh A, Pahwa M. Lethal midline granuloma. Indian Dermatol Online J. 2013;4(1):37-9. doi: 10.4103/2229-5178.105469

10. Fuller J. Selección del instrumental quirúrgico. En: Fuller J, Ness E (editores). Instrumentación quirúrgica: teoría, técnicas y procedimientos. $4 .^{\mathrm{a}}$ edición. México: Editorial Médica Panamericana; 2007. p. 457-9.

11. Zuber TJ. Fusiform excision. Am Fam Physician. 2003;67(7):1539-44, 1547-8, 1550.

12. Goldberg LH, Alam M. Elliptical excisions: variations and the eccentric parallelogram. Arch Dermatol. 2004;140(2):176-80. doi: 10.1001/archderm.140.2.176

13. Pickett H. Shave and punch biopsy for skin lesions. Am Fam Physician. 2011;84(9):995-1002.

14. Goldman G. The current status of curettage and electrodesiccation. Dermatol Clin. 2002;20(3):56978, ix. doi: 10.1016/s0733-8635(02)00022-0

15. StataCorp. 2013. Stata Statistical Software: Release 13. College Station, TX: StataCorp LP.

16. Segal N, Osyntsov L, Olchowski J, Kordeluk S, Plakht Y. Nose biopsy: a comparison between two sampling techniques. Eur Arch Otorhinolaryngol. 2016;273(6):1445-8. doi: 10.1007/s00405-015-3754-y 